\title{
Erratum to: Rapid progression of rhabdoid components of a composite high-grade glioma and rhabdoid tumor in the occipital lobe of an adult
}

\author{
Junkoh Yamamoto $\cdot$ Mayu Takahashi $\cdot$ Yoshiteru Nakano $\cdot$ Yoshiteru Soejima • \\ Takeshi Saito • Daisuke Akiba · Junko Hirato • Yoichi Nakazato • \\ Shigeru Nishizawa
}

Published online: 26 February 2012

(C) The Japan Society of Brain Tumor Pathology 2012

\section{Erratum to: Brain Tumor Pathol}

\section{DOI 10.1007/s10014-011-0069-6}

Unfortunately, errors appeared in the above-cited article, as follows:

1. In the Discussion section, in the 4th paragraph, on line 6, "Anaplastic astrocytoma" should be "Anaplastic ependymoma".

2. In the Conclusion, on lines 5 and 6, "positive INI1" should be "negative INI1".

3. In Fig. 6, references were cited in error. Regarding a case of anaplastic ependymoma reported by Judkins et al., the original diagnosis of the case was revised to AT/RT in their article cited as Ref. 9. Therefore, on reconsideration we decided that this case should be included within "Primary AT/RT". Because Perry et al. did not mention a case of leiomyosarcoma as AT/ RT in their article cited in Ref. 11, "(AT/RT)" following "Rhabdoid tumor" has been deleted. In addition, the legend for Fig. 6 has been revised as follows:
Hypothetical diagram of tumor differentiation of primary atypical teratoid/rhabdoid tumors (AT/RT), composite rhabdoid tumors (CRT), and secondary rhabdoid phenotype with respect to INII inactivation, including four controversial reports

The corrected Fig. 6 is shown on the following page.

The online version of the original article can be found under doi:10.1007/s10014-011-0069-6.

J. Yamamoto $(\bowtie) \cdot$ M. Takahashi · Y. Nakano · Y. Soejima

T. Saito $\cdot$ D. Akiba $\cdot$ S. Nishizawa

Department of Neurosurgery, University of Occupational and

Environmental Health, 1-1 Iseigaoka, Yahatanishi-ku,

Kitakyushu, Fukuoka 807-8555, Japan

e-mail: yama9218@med.uoeh-u.ac.jp

J. Hirato - Y. Nakazato

Department of Human Pathology, Gunma University Graduate

School of Medicine, Maebashi, Gunma, Japan 


\section{Pluripotent cells}

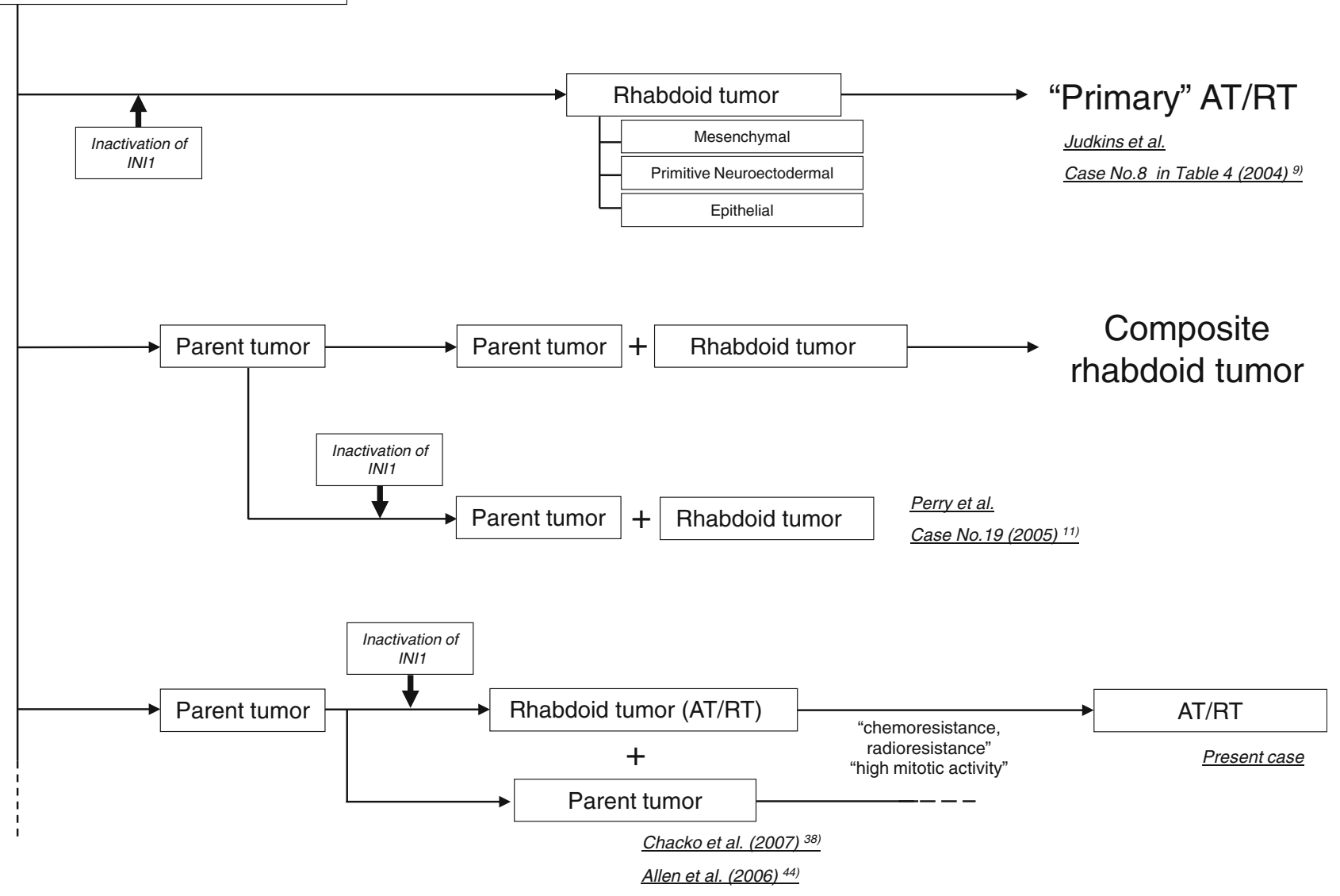

Fig. 6 Hypothetical diagram of tumor differentiation of primary atypical teratoid/rhabdoid tumors (AT/RT), composite rhabdoid tumors (CRT), and secondary rhabdoid phenotype with respect to INII inactivation, including four controversial reports 